\title{
Correction to: Sex-Specific Transcriptome Sequencing of Zoea I Larvae and Identification of Sex-Linked Genes Using Bulked Segregant Analysis in Pacific White Shrimp Litopenaeus vannamei
}

\author{
Yue Wang ${ }^{1,2,3} \cdot$ Yang Yu ${ }^{1,2,4} \cdot$ Shihao $\mathrm{Li}^{1,2,4} \cdot$ Xiaojun Zhang ${ }^{1,2,4} \cdot$ Jianhai Xiang ${ }^{1,2,4} \cdot$ Fuhua $\mathrm{Li}^{1,2,4,5}$ \\ Published online: 3 June 2021 \\ ○) Springer Science+Business Media, LLC, part of Springer Nature 2021
}

Correction to: Marine Biotechnology (2020) 22:423-432

https://doi.org/10.1007/s10126-020-09962-7

The original version of this article unfortunately contained a mistake in the Funding section. The funding number of the Joint NSFC-ISF Research Program is incorrectly presented as "3181101109" instead of "31861143047". The correct Funding information is presented below:

Funding This work is supported by the Joint NSFC-ISF Research Program (31861143047), the National Key R\&D Program of China (2018YFD0901301), the Strategic Priority Research Program of the Chinese Academy of Sciences (Grant No. XDA24030105), and the China Agriculture Research System-48.

Publisher's Note Springer Nature remains neutral with regard to jurisdictional claims in published maps and institutional affiliations.

The original article can be found online at https://doi.org/10.1007/ s10126-020-09962-7.

Yang Yu

yuyang@qdio.ac.cn

$\triangle$ Fuhua Li

fhli@qdio.ac.cn

1 Key Laboratory of Experimental Marine Biology, Institute of Oceanology, Chinese Academy of Sciences, Qingdao 266071, China

2 Laboratory for Marine Biology and Biotechnology, Qingdao National Laboratory for Marine Science and Technology, Qingdao 266000, China

3 University of Chinese Academy of Sciences, Beijing 100049, China

4 Center for Ocean Mega-Science, Chinese Academy of Sciences, Qingdao 266071, China

5 The Innovation of Seed Design, Chinese Academy of Sciences, Wuhan, People's Republic of China 